\title{
New Methods to Find Optimal Non-Disjoint Bi-Decompositions
}

\author{
Shigeru Yamashita Hiroshi Sawada Akira Nagoya \\ NTT Communication Science Laboratories \\ 2-Chome, Hikaridai, Seika-cho, Soraku-gun, Kyoto 619-02 Japan \\ Tel: +81-774-95- $\{1867,1866,1860\}$ \\ Fax: +81-774-95-1876 \\ e-mail: \{ger, sawada, nagoya\}@cslab.kecl.ntt.co.jp
}

\begin{abstract}
This paper presents new efficient methods to find "optimal bi-decomposition" forms of logic functions. An "optimal bi-decomposition" form of $f(X)$ is $f=\alpha\left(g_{1}\left(X^{1}\right), g_{2}\left(X^{2}\right)\right)$ where the total number of variables in $X^{1}$ and $X^{2}$ is the smallest among all bi-decomposition forms of $f$. We consider two methods; one's decomposition form is $\left(g_{1} \cdot g_{2}\right)$ and the other's is $\left(g_{1} \oplus g_{2}\right)$. The proposed methods can find one of the existing "optimal" decomposition forms efficiently based on the Branch-and-Bound algorithm. These methods can decompose incompletely specified functions. Preliminary experimental results show that the proposed methods can construct networks with fewer levels than conventional methods.
\end{abstract}

\section{INTRODUCTION}

When implementing a combinational logic function using a given technology, the desired function must be decomposed or factorized to smaller functions so that the decomposed functions can fit onto the implementation primitives of the technology. Many decomposition methods, therefore, have been proposed. Among them, AND/OR factoring and weak division [1] are supreme methods when expressions are in sum-of-product forms. In some cases, however, other approaches produce better results. For example, factoring with XOR can express some logic functions simpler than AND/OR factoring $[2,3]$. As for the synthesis of LUT (Look-Up Table) networks, functional decomposition [4] based methods can often produce better results [5].

Most of the previously proposed functional decomposition methods have been based on Roth-Karp decomposition [6], and thus they decompose function $f$ to the following form: $f=\alpha\left(g_{1}\left(X^{B}\right), \ldots, g_{t}\left(X^{B}\right), X^{F}\right)=$ $\alpha\left(\vec{g}\left(X^{B}\right), X^{F}\right)$, where $X^{B}$ and $X^{F}$ are sets of variables. We can think of another strategy for functional decomposition: function $f$ is decomposed into only two functions as $f=\alpha\left(g_{1}\left(X^{1}\right), g_{2}\left(X^{2}\right)\right)$, where $X^{1}$ and $X^{2}$ are sets of variables. This decomposition is called bi-decomposition [7]. If $X^{1}$ and $X^{2}$ are disjoint, the bi-decomposition form can be found very quickly [7]. In some cases, a "non- disjoint" bi-decomposition form can provide the best decomposition. (An example will be shown in Section II.)

The methods proposed in $[8,9]$ can find non-disjoint bi-decomposition forms efficiently using the notion of "groupability". The methods can find a bi-decomposition form for given $X^{1}$ and $X^{2}$, but they still have a problem selecting the best $X^{1}$ and $X^{2}$.

In this paper, we propose new efficient methods to find "optimal" non-disjoint bi-decomposition forms of incompletely specified functions. Here, "optimal" means that the total number of variables in $X^{1}$ and $X^{2}$ is the smallest among all bi-decomposition forms. This meaning is thought to be adequate for the synthesis of LUT networks, because an LUT can realize a complex function if the number of input variables does not exceed the maximum number of inputs of the LUT. We think our methods can provide a solution to the problem of how to select the best $X^{1}$ and $X^{2}$, especially in LUT network synthesis.

This paper is organized as follows. In Section II, we explain non-disjoint bi-decomposition and formulate our problem. In Section III, we propose the novel methods to find "optimal" bi-decomposition forms. We present preliminary experimental results in Section IV. Section $\mathrm{V}$ concludes this paper.

\section{Preliminaries}

\section{A. Non-Disjoint Bi-Decomposition}

The decomposition form $f=\alpha\left(g_{1}\left(X^{1}\right), g_{2}\left(X^{2}\right)\right)$ is called a bi-decomposition form [7]. If $X^{1}$ and $X^{2}$ are disjoint, it is called a "disjoint" bi-decomposition form. If $X^{1}$ and $X^{2}$ are not disjoint, it is called a "non-disjoint" bi-decomposition form.

Disjoint bi-decomposition forms are very useful for logic synthesis, and they can be found quickly [7]. However, there are functions that can be decomposed efficiently only by non-disjoint bi-decomposition. For example, suppose we want to decompose $\left(x_{1}+x_{2}+x_{3}\right) \cdot\left(x_{2} \oplus\right.$ $\left.x_{3} \oplus x_{4}\right) \cdot\left(x_{1} \oplus x_{3} \oplus x_{5}\right)$. With disjoint bi-decomposition, we cannot decompose the function. With the recursive use of non-disjoint bi-decomposition, however, we can 


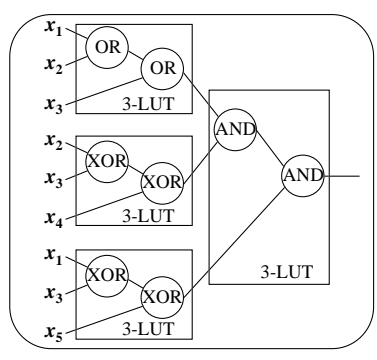

Fig. 1. An LUT Network Obtained by Non-Disjunctive Decomposition

decompose the function as shown in Fig. 1. If we want to realize the function by 3 -input LUTs, we can get an LUT network as shown in Fig. 1. This network is the same as a straightforward realization from the expression. With a Roth-Karp decomposition based method, we can not find such a good decomposition form of this example.

\section{B. Problem Formulation}

In LUT network synthesis, one of the costs of a function is the number of variables which the function depends on. Therefore, we define an "optimal" bi-decomposition form as follows:

Definition $1 f(X)=\alpha\left(g_{1}\left(X^{1}\right), g_{2}\left(X^{2}\right)\right)$ is called an "optimal" bi-decomposition form if the total number of variables in $X^{1}$ and $X^{2}$ is the smallest among all bidecomposition forms of $f$.

If $X^{1}$ (or $X^{2}$ ) is an empty set, the decomposition is a trivial decomposition, which we ignore in this paper. For example, $f(X)=1 \cdot f(X)$ is a trivial decomposition, and $X^{1}$ is an empty set. Even if the total number of variables in $X^{1}$ and $X^{2}$ of a trivial decomposition form is the smallest among all bi-decomposition forms, the trivial decomposition is not called optimal. Note that an optimal bi-decomposition of a function may be disjoint or nondisjoint depending on the function, and there may be no optimal bi-decomposition forms for some functions.

To find a bi-decomposition form $\alpha\left(g_{1}\left(X^{1}\right), g_{2}\left(X^{2}\right)\right)$, we need to consider only three decomposition forms: $\left(g_{1}\left(X^{1}\right) \cdot g_{2}\left(X^{2}\right)\right),\left(g_{1}\left(X^{1}\right)+g_{2}\left(X^{2}\right)\right)$, and $\left(g_{1}\left(X^{1}\right) \oplus\right.$ $\left.g_{2}\left(X^{2}\right)\right)$, which we call the "AND-Decomposition", "OR-Decomposition", and "XOR-Decomposition" forms, respectively. Since $f$ can be decomposed into an OR-Decomposition form iff $\bar{f}$ can be decomposed into an $A N D$-Decomposition form, we only consider $A N D$ Decomposition and XOR-Decomposition in this paper.

For an incompletely specified function $f$, the goals of this paper are:

1. Finding an optimal AND-Decomposition form, and

2. Finding an optimal XOR-Decomposition form.

Although there may be more than two optimal bidecomposition forms, our problem is to find just one of them.

\section{Optimal Non-Disjoint Bi-Decompositions}

In this section, we present efficient methods to find optimal AND-Decomposition and XOR-Decomposition forms.

\section{A. Optimal AND-Decomposition}

Here, we present a method to decompose incompletely specified function $f$ into an optimal bi-decomposition form: $f(X)=g_{1}(X) \cdot g_{2}(X)$.

First we generate an initial solution $\left(g_{1}, g_{2}\right)$ as will be mentioned later. From the initial solution, the recursive procedure "DecompAND" shown in Fig. 2 improves the solution to produce an optimal $A N D$-Decomposition form of $f$ based on the Branch-and-Bound algorithm. Although there may be more than two optimal solutions according to our definition in Section II, the procedure only finds one of them.

\section{A.1 Definitions for DecompAND}

In the procedure DecompAND, $g_{1}$ and $g_{2}$ are treated as four-valued functions whose values are $0,1, * 0$ or $*$. * means a usual don't care. $* 0$, which is introduced in this paper, means that $g_{1}\left(g_{2}\right)$ can be treated as a usual don't care if $g_{2}\left(g_{1}\right)$ is treated as 0 , and $g_{1}\left(g_{2}\right)$ must be 0 if $g_{2}$ $\left(g_{1}\right)$ is treated as 1 . In other words, $* 0$ means that at least one of the functions must be 0 . The following condition is maintained throughout DecompAND.

- $g_{1}(a)=* 0$ iff $g_{2}(a)=* 0$. ( $a$ is a minterm. $)$

This conditions means that if we change $g_{1}$ so that $\left(g_{1}(a)=* 0\right)$ is changed to $\left(g_{1}(a)=1\right)$, we also change $g_{2}$ so that $\left(g_{2}(a)=* 0\right)$ is changed to $\left(g_{2}(a)=0\right)$, and if we change $g_{1}$ so that $\left(g_{1}(a)=* 0\right)$ is changed to $\left(g_{1}(a)=0\right)$, we also change $g_{2}$ so that $\left(g_{2}(a)=* 0\right)$ is changed to $\left(g_{2}(a)=*\right)$. The introduction of $* 0$ makes it possible to find an optimal solution based on the Branch-andBound algorithm. The following definitions of functions and operations are used in our method. In the definitions, $g$ is a four-valued function, $f$ is an incompletely specified function (three-valued) or a four-valued function, and $h$ is a completely specified function (two-valued).

- $O N(f)$ means a characteristic function that represents a set of minterms $\{a \mid f(a)=1\}$.

- $O F F(f)$ means a characteristic function that represents a set of minterms $\{a \mid f(a)=0\}$.

- $D C 0(g)$ means a characteristic function that represents a set of minterms $\{a \mid g(a)=* 0\}$.

- $D C(f)$ means a characteristic function that represents a set of minterms $\{a \mid f(a)=*\}$.

- $h_{x_{i}}$ and $h_{\bar{x}_{i}}$ mean the positive and negative cofactors of $h$ with respect to $x_{i}$, respectively. 


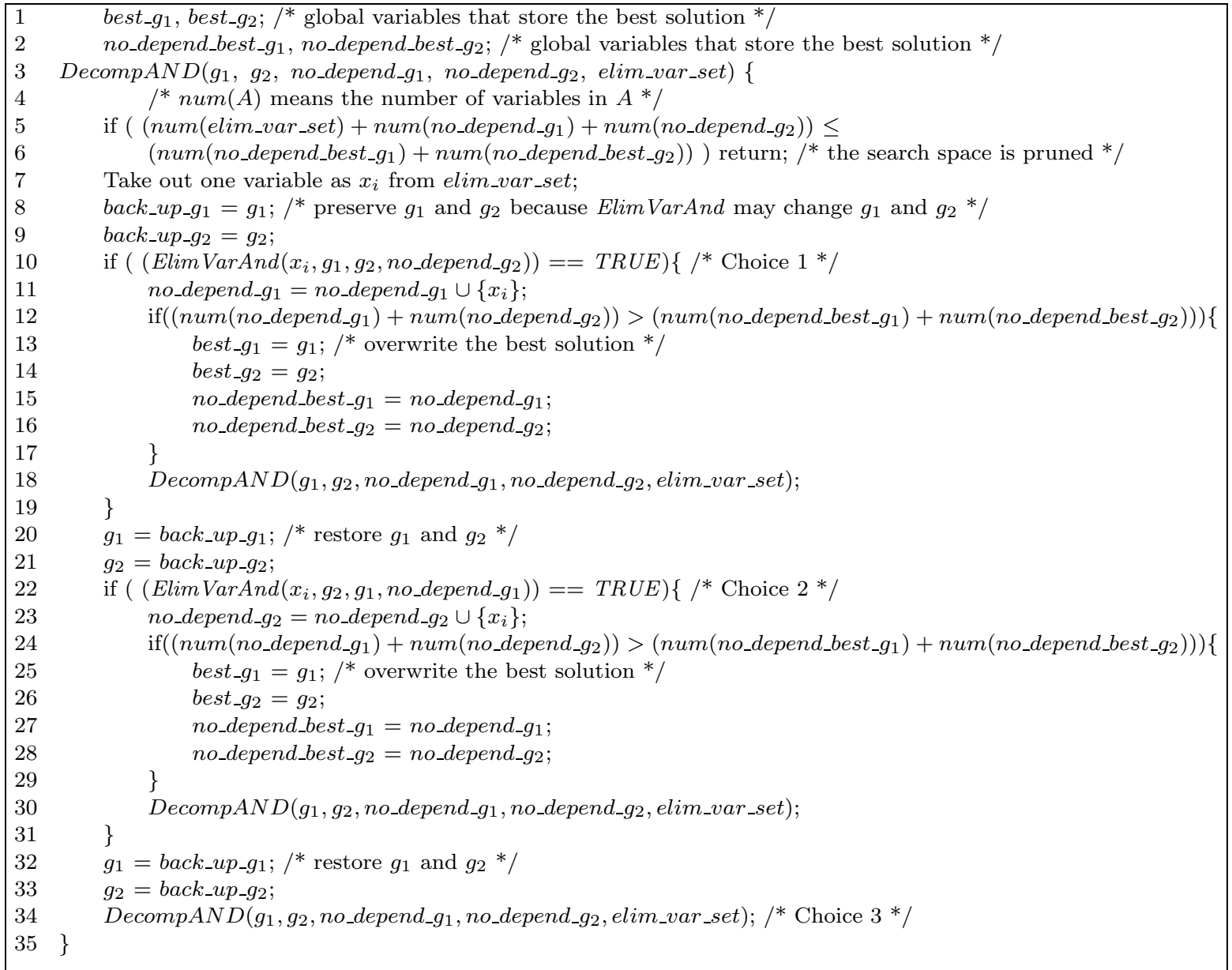

Fig. 2. DecompAND

- $\operatorname{Smooth}\left(x_{i}, h\right)$ means $h_{x_{i}}+h_{\bar{x}_{i}}$.

- SmoothSet(var_set,h) means a function that is obtained by applying successively $\operatorname{Smooth}\left(x_{i}, h\right)$ to $h$ for all $x_{i}$ in var_set.

- EnlargeON $(g, h)$ means an operation to change $g$ so that $O N(g)$ is changed to $O N(g)+h$. (Note that $O F F(g), D C 0(g)$ and $D C(g)$ must be changed to proper functions at the same time. Similar operations are also needed at EnlargeOFF $(g, h)$ and Enlarge $D C(g, h)$.

- EnlargeOFF $(g, h)$ means an operation to change $g$ so that $O F F(g)$ is changed to $O F F(g)+h$.

- Enlarge $D C(g, h)$ means an operation to change $g$ so that $D C(g)$ is changed to $D C(g)+h$.

Note that $O N(g), O F F(g), D C 0(g)$ and $D C(g)$ are completely specified functions, whereas $g$ is a four-valued function.

The definitions of the variables used in DecompAND are as follows.
- best_ $g_{1}$ and best_ $g_{2}$ represent $g_{1}$ and $g_{2}$ in an optimal solution, respectively.

- no_depend_(function) represents a set of variables that have already been eliminated explicitly from the dependency of function.

- elim_var_set represents a set of variables that have the possibility of being eliminated from the dependency of $g_{1}$ or $g_{2}$.

\section{A.2 DecompAND}

Now, we explain the procedure Decomp $A N D$ using an example. Suppose we want to find an optimal $A N D$ Decomposition of function $f$ : the ON-set is $\left(x_{2}+\bar{x}_{4}\right) \cdot\left(x_{1}\right.$. $\left.x_{4}+\bar{x}_{1} \cdot \bar{x}_{3}\right)$ and the DC-set is $x_{3} \cdot\left(x_{2} \cdot \bar{x}_{4}+x_{1} \cdot \bar{x}_{2} \cdot x_{4}\right)$. The truth table of $f$ is shown at the top left-hand corner of Fig. 3. At first best_g $g_{1}$ and best_g $g_{2}$ are set to null. no_depend_best_g $g_{1}$ and no_depend_best_g $g_{2}$ are set to $\emptyset$. elim_var_set is set to the set of variables which $f$ depends on. In the example, it is set to $\left\{x_{1}, x_{2}, x_{3}, x_{4}\right\}$. Initial $g_{1}$ ( $g_{2}$ is the same) is produced to satisfy $O N\left(g_{1}\right)=O N(f)$, $D C 0\left(g_{1}\right)=O F F(f)$, and $D C\left(g_{1}\right)=D C(f)$. Clearly, 


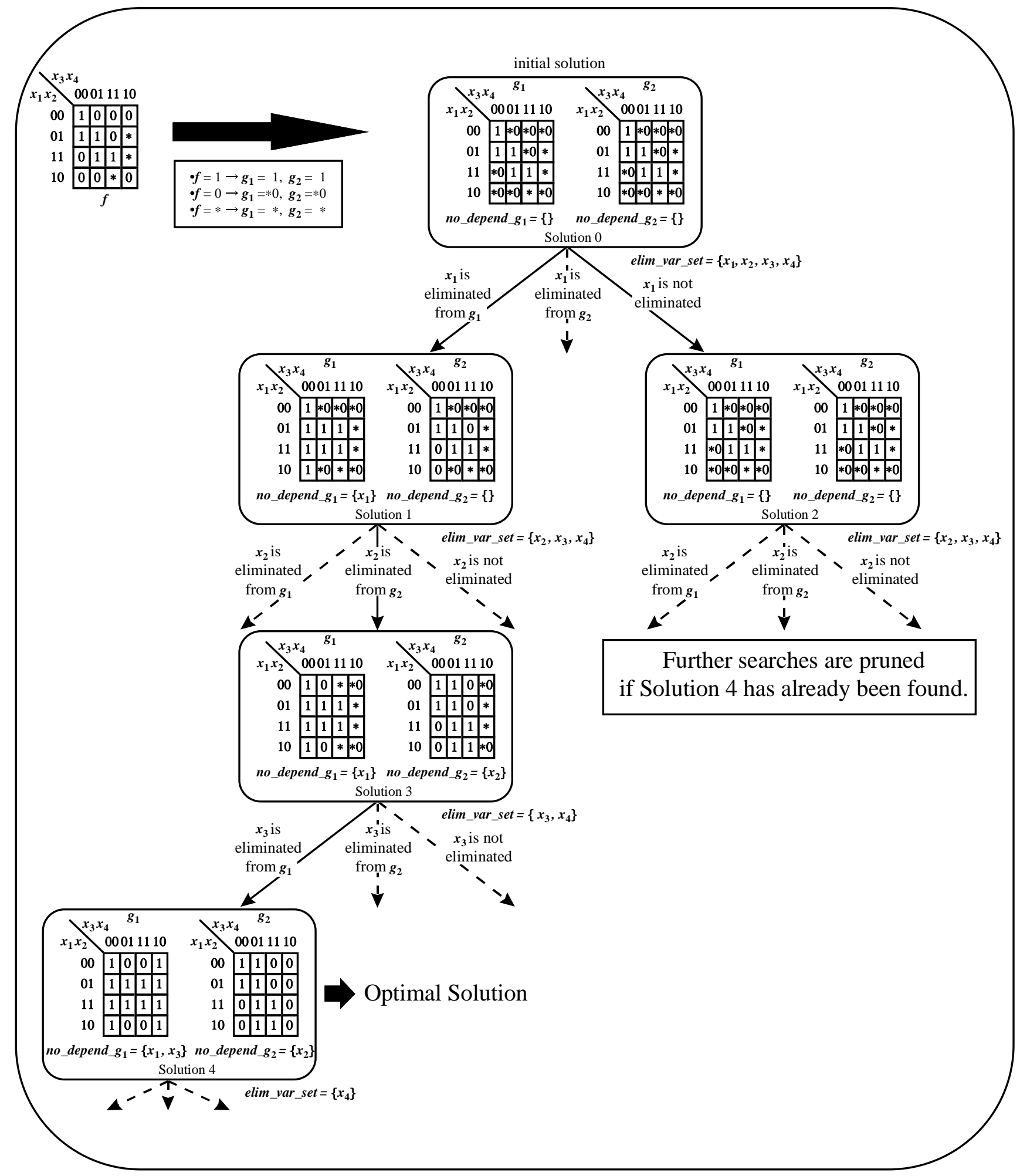

Fig. 3. A Search Tree for Finding an Optimal AND-Decomposition 
this initial solution satisfies $f(X)=g_{1}(X) \cdot g_{2}(X)$. In the example, the initial solution is shown as "Solution 0 " in Fig. 3. In the initial solution, no_depend $d_{-} g_{1}$ and $n o_{-} d e p e n d_{-} g_{2}$ are $\emptyset$. Initial $g_{1}$ and $g_{2}$ have $* \mathrm{~s}$ and $* 0 \mathrm{~s}$. By specifying these values to 1 or $0, D e c o m p A N D$ eliminates variables from the dependency of $g_{1}$ or $g_{2}$. The search tree of the example is shown in Fig. 3. For instance, from "Solution 0" to "Solution 1" $x_{1}$ is eliminated from the dependency of $g_{1}$, and $x_{1}$ is added to no_depend $d_{-} g_{1}$. If $x_{1}$ is eliminated from the dependency of $g_{1}, x_{1}$ cannot be eliminated from the dependency of $g_{2}$, because $f$ depends on $x_{1}$. Therefore, when $x_{1}$ is eliminated from the dependency of $g_{1}$, we delete $x_{1}$ from elim_var_set.

From an intermediate solution, we have three choices to search further:

Choice 1 We eliminate a variable $\left(x_{i}\right)$ in elim_var_set from the dependency of $g_{1}$, and then search further. (This corresponds to line 18 in Fig. 2 and the left arrow from each solution in Fig. 3.)

Choice 2 We eliminate a variable $\left(x_{i}\right)$ in elim_var_set from the dependency of $g_{2}$, and then search further. (This corresponds to line 30 in Fig. 2 and the middle arrow from each solution in Fig. 3.)

Choice 3 We do not eliminate a variable $\left(x_{i}\right)$ in elim_var_set from the dependency of either $g_{1}$ and $g_{2}$, and then search further. (This corresponds to line 34 in Fig. 2 and the right arrow from each solution in Fig. 3.)

If we select Choice 1 or 2 , we must eliminate $x_{i}$ from the dependency of $g_{1}$ or $g_{2}$, respectively. This is done by the procedure Elim VarAnd which is mentioned in the next section.

If we successfully eliminate a variable from an intermediate solution, and a new solution is better than the best solution: (best $g_{1}$, best $_{-} g_{2}$ ), we overwrite the best solution at lines 13 to 16 , and 25 to 28 in Fig. 2 .

Let us consider when a search is pruned. For example, if "Solution 4" in Fig. 3 has already been found, we do not need to search further from "Solution 2". The reason is as follows. The total number of variables in no_depend $g_{1}$ and no_depend g $_{2}$ of "Solution 4" and that of "Solution 2 " are three and zero, respectively. From "Solution 2", we can eliminate three variables from the dependency of $g_{1}$ or $g_{2}$ at best because the number of variables in elim_var_set of "Solution 2" is three. Therefore, we cannot find a better solution than "Solution 4" in the search space from "Solution 2". This check is done at lines 5 and 6 in Fig. 2.

\section{A.3 Elim VarAnd}

The procedure Elim VarAnd $\left(x_{i}, g_{1}, g_{2}\right.$, no_depend_ $\left.g_{2}\right)$ used in DecompAND eliminates $x_{i}$ from the dependency of $g_{1}$. This is shown in Fig. 4. Of course, the following conditions must be kept throughout the operations of Elim VarAnd.

Condition $1 f=g_{1} \cdot g_{2}$.

Condition $2 g_{1}$ does not depend on the variables in $n o_{-} d e p e n d_{-} g_{1}$.

Condition $3 g_{2}$ does not depend on the variables in no_depend $g_{2}$.

For example, from "Solution 3" in Fig. 3, $x_{3}$ is eliminated from the dependency of $g_{1}$ to get "Solution 4" as follows. At first we calculate what parts of $D C\left(g_{1}\right)$ and $D C 0\left(g_{1}\right)$ must be changed to $O N\left(g_{1}\right)$ so that $g_{1}$ does not depend on $x_{i}$. This calculation corresponds to change $1_{-} g_{1}$ at line 5 in Fig. 4 . Next, we calculate what parts of $D C\left(g_{1}\right)$ and $D C 0\left(g_{1}\right)$ must be changed to $O F F\left(g_{1}\right)$ so that $g_{1}$ does not depend on $x_{i}$. This calculation corresponds to change $0_{-} g_{1}$ at line 6 in Fig. 4. In the example, these functions are calculated as "change $1_{-} g_{1}$ " and "change $0_{-} g_{1}$ " in Fig. 5, respectively.

Then, we change $g_{1}$ by EnlargeON $\left(g_{1}\right.$, change $\left.1_{-} g_{1}\right)$ and EnlargeOFF $\left(g_{1}\right.$, change $\left.0_{-} g_{1}\right)$ at lines 13 and 14 in Fig. 4. Although we change $g_{1}$ by the above operations, we cannot eliminate $x_{i}$ from the dependency of $g_{1}$ when $\left(\operatorname{Smooth}\left(x_{i}, O N\left(g_{1}\right)\right) \cdot \operatorname{Smooth}\left(x_{i}, O F F\left(g_{1}\right)\right)\right)$ is not the constant 0 function. This is checked at line 3 in Fig. 4, and FALSE is returned if $x_{i}$ cannot be eliminated. For example, we cannot eliminate $x_{4}$ from $g_{1}$ of "Solution 3" in Fig. 3. This is because $\operatorname{Smooth}\left(x_{4}, O N\left(g_{1}\right)\right)$. $\operatorname{Smooth}\left(x_{4}, O F F\left(g_{1}\right)\right.$ ) (shown as "obstacle" in Fig. 5) is not the constant 0 function. Therefore, ElimVarAnd returns FALSE in this case. Indeed the parts of $O N\left(g_{1}\right)$ and $O F F\left(g_{1}\right)$ masked by $O N($ "obstacle") make it impossible to eliminate $x_{4}$ from the dependency of $g_{1}$ of "Solution 3".

After these operations, $g_{1}$ is changed to a function which does not depend on $x_{3}$. This function is shown as "new $g_{1}$ " in Fig. 5. However, remember that $* 0$ is not a usual don't care, and therefore, we need some more operations as follows to satisfy Condition 1. If a part of $D C 0\left(g_{1}\right)$ is changed to $O N\left(g_{1}\right)$ by EnlargeON $\left(g_{1}\right.$, change $\left.1_{-} g_{1}\right)$, the corresponding part of $D C 0\left(g_{2}\right)$ must be changed to $O F F\left(g_{2}\right)$. The corresponding part of $D C 0\left(g_{2}\right)$ is calculated as must_change $0_{-} g_{2}=$ change $1_{-} g_{1} \cdot D C 0\left(g_{1}\right)$ (at line 8 in Fig. 4). This function is shown as "first must_change $0_{-} g_{2}$ " in Fig. 5. Therefore, EnlargeOFF $\left(g_{2}\right.$, must_change $\left.0_{-} g_{2}\right)$ at line 15 in Fig. 4 is needed.

By the operations mentioned above, we can get a new solution: (new_ $g_{1}$, half-finished $\left.n e w_{-} g_{2}\right)$, where $n e w_{-} g_{1}$ does not depend on $x_{3}$ and the solution satisfies Condition 1. new_ $g_{1}$ and half-finished $n e w_{-} g_{2}$ are shown in Fig. 5. Here, let us check Conditions 2 and 3. Since $g_{1}$ does not depend on the variables in no_depend_ $g_{1}$, change $1_{-} g_{1}$ and change $0_{-} g_{1}$ do 


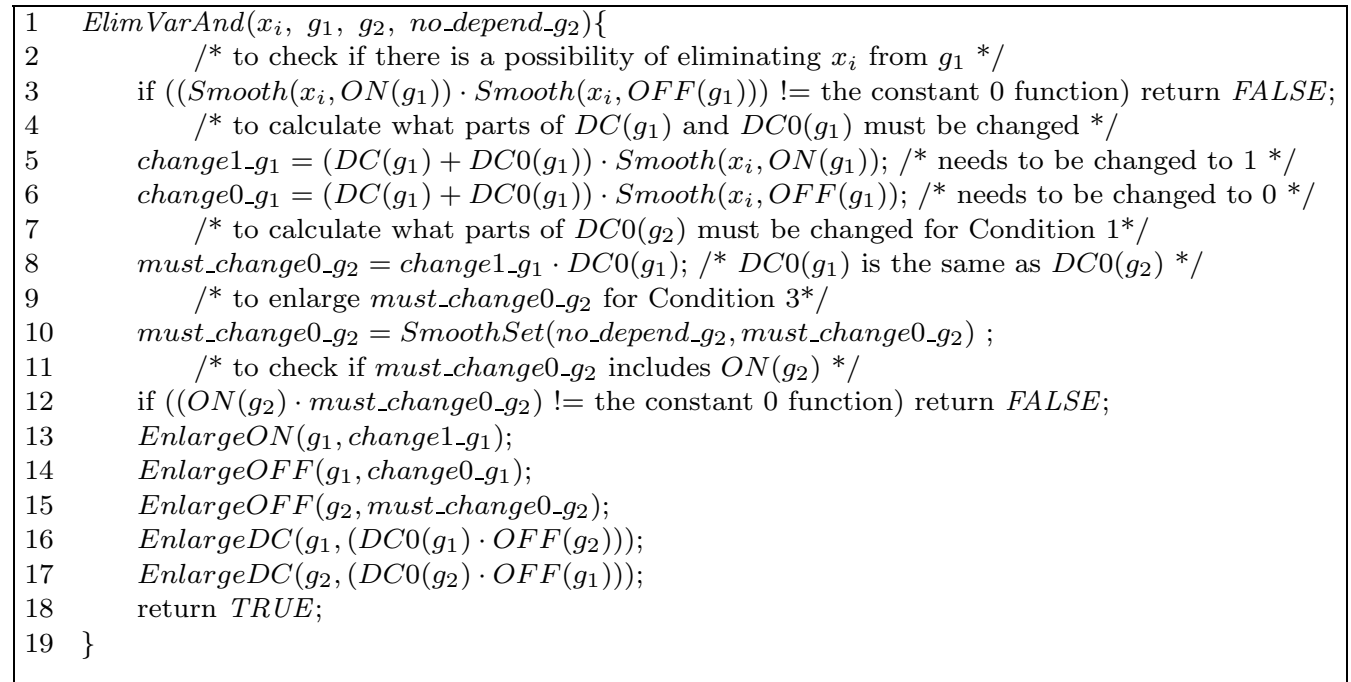

Fig. 4. Elim VarAnd

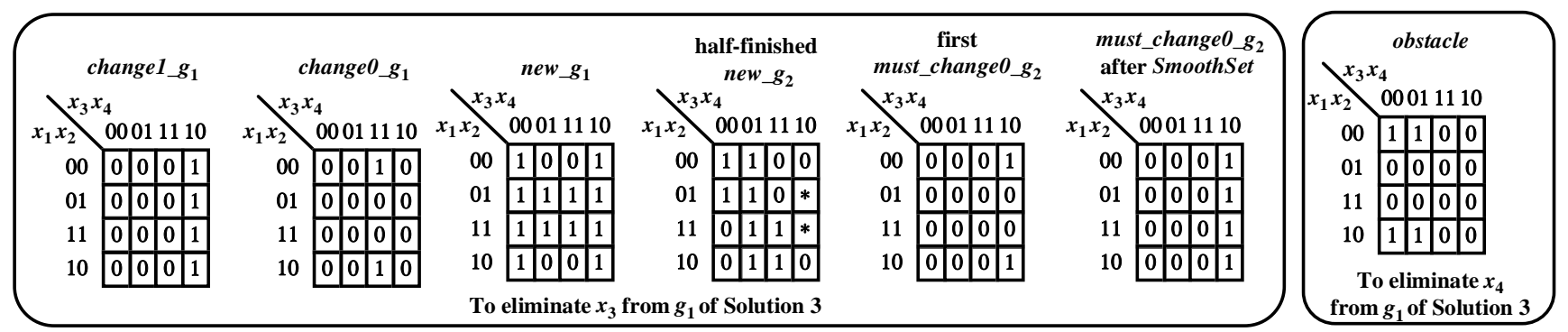

Fig. 5. Functions to Explain Elim VarAnd

not depend on the variables in no_depend $g_{1}$. Therefore, $n e w_{-} g_{1}$ still does not depend on the variables in $n_{0}{ }_{d e p e n d} g_{1}$ after Enlarge $O N\left(g_{1}\right.$, change $\left.1_{-} g_{1}\right)$ and EnlargeOFF $\left(g_{1}\right.$, change $\left.0_{-} g_{1}\right)$, that is, Condition 2 is satisfied. However, Condition 3 is not satisfied because there is no guarantee that must_change $0_{-} g_{2}$ does not depend on the variables in no_depend_ $g_{2}$. In the example, must_change0_ $g_{2}$ (see "first must_change0_ $g_{2}$ " in Fig. 5) and half-finished $n e w_{-} g_{2}$ indeed depend on $x_{2}$ which is a variable in no_depend $d_{-} g_{2}$. Thus, if we need to change a part of $D C 0\left(g_{2}\right)$ to $O F F\left(g_{2}\right)$, we must also change the corresponding part obtained by SmoothSet(no_depend_ $g_{2}$, the part) to $O F F\left(g_{2}\right)$ so that $g_{2}$ does not depend on the variables in no_depend $g_{2}$. In the example, SmoothSet(no_depend_g $g_{2}$, must_change0_-g $g_{2}$ ) is calculated as "must_change0_ $g_{2}$ after SmoothSet" in Fig. 5. Therefore, the calculation at line 10 in Fig. 4 is needed.

In some cases, must_change $0_{-} g_{2}$ after SmoothSet is a function that includes a part of $O N\left(g_{2}\right)$. In such cases, we cannot do EnlargeOFF $\left(g_{2}\right.$, must_change0_ $\left.g_{2}\right)$ at line 15 in Fig. 4 while keeping Condition 1. Therefore, in such cases, $x_{i}$ cannot be eliminated from $g_{1}$ while keeping the above three conditions, and ElimVarAnd returns FALSE (at line 12 in Fig. 4). If during the above operations a part of $D C 0\left(g_{1}\right)\left(D C 0\left(g_{2}\right)\right)$ is changed to $O F F\left(g_{1}\right)\left(O F F\left(g_{2}\right)\right)$, the corresponding part of $D C 0\left(g_{2}\right)$ $\left(D C 0\left(g_{1}\right)\right)$ can be changed to don't care (usual don't care). Therefore, we can enlarge the don't cares of $g_{1}$ and $g_{2}$ by EnlargeDC $\left(g_{1},\left(D C 0\left(g_{1}\right) \cdot O F F\left(g_{2}\right)\right)\right)$ at line 16 in Fig. 4 and Enlarge $D C\left(g_{2},\left(D C 0\left(g_{2}\right) \cdot O F F\left(g_{1}\right)\right)\right)$ at line 17 in Fig. 4, respectively. In the example, finally we get $g_{1}$ and $g_{2}$ of "Solution 4" in Fig. 3 .

In this way, Decomp $A N D$ eliminates variables one by one from the dependency of $g_{1}$ and $g_{2}$ to find an optimal $A N D$-Decomposition. In the example, "Solution 4" is an optimal AND-Decomposition where $g_{1}=\left(x_{2}+\bar{x}_{4}\right)$ and $g_{2}=\left(x_{1} \cdot x_{4}+\bar{x}_{1} \cdot \bar{x}_{3}\right)$.

It is clear that DecompAND can find one of the existing optimal solutions by elimination of any order of variables. The order only affects the execution time and which solution is found among the optimal solutions if there are more than two optimal solutions.

\section{B. Optimal XOR-Decomposition}

Here, we present a method to decompose incompletely 


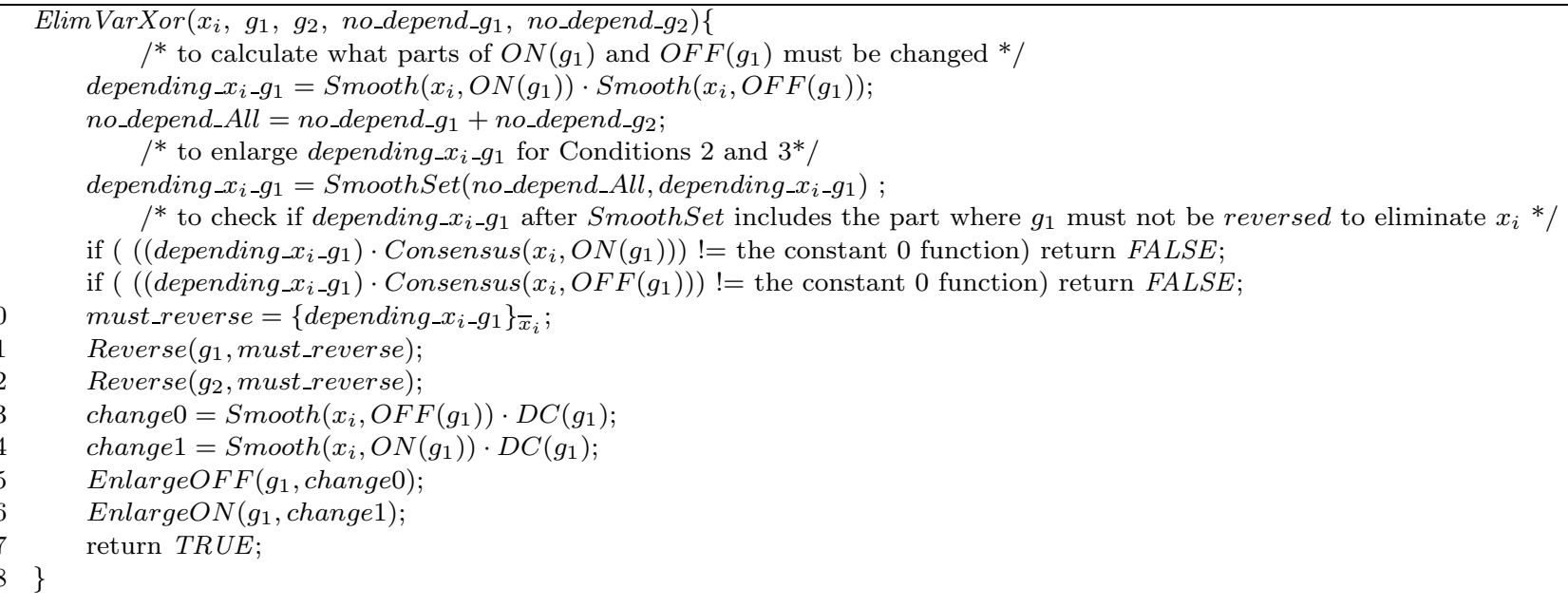

Fig. 6. Elim VarXor

specified function $f$ into an optimal bi-decomposition form: $f(X)=g_{1}(X) \oplus g_{2}(X)$. The whole procedure is called DecompXOR, which is almost the same as DecompAND. The different points are as follows:

- How to eliminate the variable dependency from $g_{1}$ and $g_{2}$ is different. Therefore, in DecompXOR, ElimVarAnd is replaced with "ElimVarXor" (mentioned later).

- In Elim VarXor, we do not use $* 0$ but only $*$ (usual don't care). Therefore, in DecompXOR, $g_{1}$ and $g_{2}$ are usual incompletely specified functions (three-valued).

- The initial solution is different. Initial $g_{1}$ is the same as $f$. Initial $g_{2}$ is the constant 0 function with the same don't cares as $f$. Clearly, this initial solution satisfies $f(X)=g_{1}(X) \oplus g_{2}(X)$.

From the initial solution, we eliminate the variable dependency from $g_{1}$ or $g_{2}$ by ElimVarXor in Fig. 6 . We explain procedure DecompXOR using an example. Suppose we want to find an optimal XOR-Decomposition of function $f$ : the ON-set is $\left(x_{2}+\bar{x}_{4}\right) \oplus\left(x_{1} \cdot x_{4}+\bar{x}_{1} \cdot \bar{x}_{3}\right)$ and the DC-set is $x_{3} \cdot\left(x_{2} \cdot \bar{x}_{4}+\bar{x}_{1} \cdot \bar{x}_{2} \cdot x_{4}\right)$. The truth table of $f$ is shown at the top left-hand corner of Fig. 7. The search tree of the example is shown in Fig. 7. The following additional definitions of a function and an operation are used in procedure Elim VarXor.

- $C o n s e n s u s\left(x_{i}, h\right)$ means $h_{x_{i}} \cdot h_{\bar{x}_{i}}$.

- Reverse $(g, h)$ means an operation to reverse the values of $g$ 's part which is masked by $O N(h)$. (reverse is an operation to change 1 to 0,0 to 1 , and $*$ to $*$.)

Here, we explain procedure ElimVarXor. Of course, the following conditions must be kept throughout the operations of Elim VarXor.
Condition $1 f=g_{1} \oplus g_{2}$.

Condition $2 g_{1}$ does not depend on the variables in $n o \_d e p e n d_{-} g_{1}$.

Condition $3 g_{2}$ does not depend on the variables in $n o \_d e p e n d_{-} g_{2}$.

To satisfy Condition 1, we can only do a pair of operations: Reverse $\left(g_{1}, h\right)$ and Reverse $\left(g_{2}, h\right)$. This is because although we reverse the values of some parts of $g_{1},\left(g_{1} \oplus g_{2}\right)$ does not change if the values of the same parts of $g_{2}$ are also reversed. Of course, we can freely change $* \mathrm{~s}$ of $g_{1}$ and $g_{2}$ to 1 or 0 .

At first, we calculate what parts of $O N\left(g_{1}\right)$ and $O F F\left(g_{1}\right)$ cause $g_{1}$ to depend on $x_{i}$. This calculation corresponds to depending $\_x_{i-} g_{1}$ at line 3 in Fig. $6 . x_{i}$ can be eliminated from the dependency of $g_{1}$, if we do Reverse ( $g_{1}$, must_reverse), where must_reverse satisfies the following two conditions which we call "Conditions for must_reverse":

- $\operatorname{Smooth}\left(x_{i}\right.$, must_reverse $)=$ depending $x_{i-} g_{1}$.

- Consensus $\left(x_{i}\right.$, must_reverse $)=$ the constant 0 function.

There are many candidates for must_reverse that satisfy the "Conditions for must_reverse". Among them we chose $\left\{\text { depending } x_{i_{-}} g_{1}\right\}_{\bar{x}_{i}}$ at line 10 in Fig. 6. For example, when we eliminate $x_{3}$ from the dependency of $g_{1}$ of "Solution 3" in Fig. 7, depending $x_{3}{ }_{3} g_{1}$ and \{depending $\left.\__{3} g_{1}\right\}_{\bar{x}_{3}}$ are calculated as shown in Fig. 8 . Then, we change $g_{1}$ and $g_{2}$ by Reverse $\left(g_{1}\right.$, must_reverse) and Reverse $\left(g_{2}\right.$, must_reverse $)$ at lines 11 and 12 in Fig. 6. To add to these operations, we must change a part of $D C\left(g_{1}\right)$ to $O N\left(g_{1}\right)$ or $O F F\left(g_{1}\right)$ so that $g_{1}$ does not depend on $x_{i}$. This is done at lines 13 to 16 in Fig. 6 . For example, when we eliminate $x_{3}$ from the dependency 


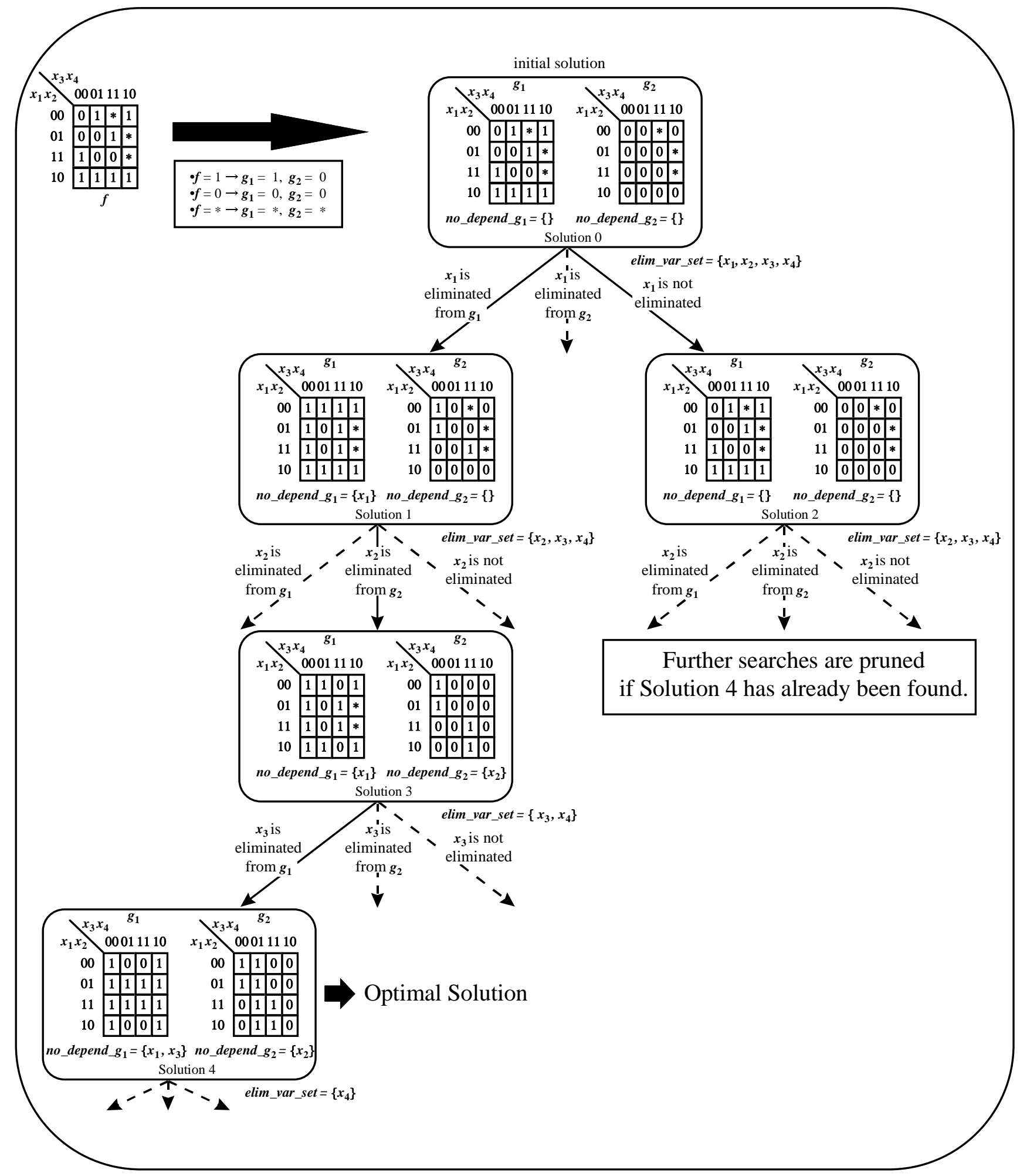

Fig. 7. A Search Tree for Finding an Optimal XOR-Decomposition 


\begin{tabular}{|c|c|c|c|c|c|c|c|c|c|c|c|c|c|c|c|c|c|c|c|c|c|c|c|c|c|c|c|c|c|c|}
\hline \multicolumn{4}{|c|}{$\begin{array}{l}\text { depending_ } x_{3} g_{1} \\
x_{1} x_{2} x_{3} x_{4} \\
\end{array}$} & \multicolumn{4}{|c|}{$\begin{array}{c}\left\{\text { depending } \_x_{3} g_{1}\right\}_{x_{3}} \\
x_{1} x_{2} x_{3} x_{4} \\
00011110\end{array}$} & \multicolumn{3}{|c|}{$\begin{array}{l}\text { change1 } \\
x_{4} \\
00011110\end{array}$} & \multicolumn{4}{|c|}{$\begin{array}{l}\text { depending_ } x_{2} \_g_{2} \\
x_{1} x_{2} x_{3} x_{4} \\
00011110\end{array}$} & \multicolumn{4}{|c|}{$\begin{array}{l}\text { depending_ } x_{2} \_g_{2} \\
\text { after SmoothSet } \\
x_{3} x_{4} \\
c_{1} x_{2} 00011110\end{array}$} & \multicolumn{4}{|c|}{$\begin{array}{l}\text { must_reverse } \\
(\text { Solution 1) } \\
x_{3} x_{4} \\
x_{2} 00011110\end{array}$} & \multicolumn{8}{|c|}{ 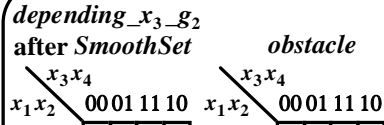 } \\
\hline 00 & \begin{tabular}{l|l}
0 & 1 \\
\end{tabular} & 1 & 10 & 00 & \begin{tabular}{l|l}
0 & 1 \\
\end{tabular} & \begin{tabular}{l|l}
1 & 0
\end{tabular} & 0 & 00 & \begin{tabular}{|l|l|}
0 & 0 \\
\end{tabular} & \begin{tabular}{|l|l|}
0 & 0 \\
\end{tabular} & 00 & 0 & 0 & 0 & 00 & \begin{tabular}{|l|l}
0 & \\
\end{tabular} & 0 & 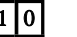 & 00 & \begin{tabular}{|l|l|}
0 & 0 \\
\end{tabular} & & 0 & 00 & \begin{tabular}{|l|l}
1 & \\
\end{tabular} & \begin{tabular}{l|l}
0 & 0 \\
\end{tabular} & \begin{tabular}{|l|l|}
0 & 1 \\
\end{tabular} & 00 & \begin{tabular}{|l|l|}
0 & 0 \\
\end{tabular} & 0 & 0 \\
\hline 01 & \begin{tabular}{l|l}
0 & 1 \\
\end{tabular} & $1 \mid 1$ & 10 & 01 & \begin{tabular}{l|l}
0 & 1 \\
\end{tabular} & \begin{tabular}{l|l}
1 & 0 \\
\end{tabular} & 0 & 01 & \begin{tabular}{|l|l|}
0 & 0 \\
\end{tabular} & \begin{tabular}{|l|l|}
0 & 1 \\
\end{tabular} & 01 & 0 & 0 & 0 & 01 & \begin{tabular}{|l|l}
0 & \\
\end{tabular} & 1 & \begin{tabular}{|l|l|}
1 & 0 \\
\end{tabular} & 01 & \begin{tabular}{|l|l}
0 & 0 \\
\end{tabular} & & 0 & 01 & \begin{tabular}{|l|l|}
0 & \\
\end{tabular} & \begin{tabular}{l|l}
1 & 1 \\
\end{tabular} & \begin{tabular}{|l|l|}
1 & 0 \\
\end{tabular} & 01 & \begin{tabular}{|l|l|}
0 & 1 \\
\end{tabular} & 1 & 0 \\
\hline 11 & \begin{tabular}{l|l}
0 & 1 \\
\end{tabular} & 11 & 10 & 11 & \begin{tabular}{l|l}
0 & 1 \\
\end{tabular} & $\begin{array}{ll}1 & 0 \\
\end{array}$ & 0 & 11 & \begin{tabular}{|l|l|}
0 & 0 \\
\end{tabular} & \begin{tabular}{|l|l|}
0 & 1 \\
\end{tabular} & 11 & 0 & j & 0 & 11 & $\begin{array}{ll}0 & \\
\end{array}$ & 1 & \begin{tabular}{l|l|}
1 & 0 \\
\end{tabular} & 11 & \begin{tabular}{l|l}
0 & 0 \\
\end{tabular} & & 0 & 11 & \begin{tabular}{|l|l|}
0 \\
\end{tabular} & \begin{tabular}{l|l}
1 & 1 \\
\end{tabular} & \begin{tabular}{ll|}
1 & 0 \\
\end{tabular} & 11 & \begin{tabular}{|l|l}
0 & 0 \\
\end{tabular} & 0 & 0 \\
\hline 10 & \begin{tabular}{l|l}
0 & 1 \\
\end{tabular} & 11 & 10 & 10 & \begin{tabular}{l|l}
0 & 1 \\
\end{tabular} & $\begin{array}{ll}1 & 0 \\
\end{array}$ & 0 & 10 & \begin{tabular}{|l|l|}
0 & 0 \\
\end{tabular} & \begin{tabular}{|l|l|}
0 & 0 \\
\end{tabular} & 10 & 0 & & 0 & 10 & \begin{tabular}{|l|l}
0 & \\
\end{tabular} & 1 & \begin{tabular}{l|l|}
1 & 0 \\
\end{tabular} & 10 & \begin{tabular}{l|l}
0 & 0 \\
\end{tabular} & & 0 & 10 & \begin{tabular}{|l|l|}
1 & \\
\end{tabular} & 0 C & \begin{tabular}{|l|l|}
0 & 1 \\
\end{tabular} & 10 & \begin{tabular}{|l|l}
1 & 0 \\
\end{tabular} & & 1 \\
\hline \multicolumn{11}{|c|}{ To eliminate $x_{3}$ from $g_{1}$ of Solution 3} & \multicolumn{12}{|c|}{ To eliminate $x_{2}$ from $g_{2}$ of Solution 1} & \multicolumn{8}{|c|}{ To eliminate $x_{3}$ from $g_{2}$ of Solution 1} \\
\hline
\end{tabular}

Fig. 8. Functions to Explain Elim VarXor

of $g_{1}$ of "Solution 3" in Fig. 7, change0 is the constant 0 function and change 1 is calculated as shown in Fig. 8. After these operations we get "Solution 4" in Fig. 7, which is an optimal solution in the example.

For Conditions 2 and 3, must_reverse must not depend on the variables in no_depend $d_{-} g_{1}$ and no_depend $d_{-} g_{2}$. Therefore, line 6 in Fig. 6 is needed. For example, when we eliminate $x_{2}$ from the dependency of $g_{2}$ of "Solution 1 " in Fig. 7, depending $x_{2} g_{2}$ at line 3 in Fig. 6 is calculated as "depending $x_{2} g_{2}$ " in Fig. 8. This function depends on $x_{1}$, and therefore, we must Smooth this function with respect to $x_{1}$ and get depending $x_{2-} g_{2}$ at line 6 in Fig. 6 as "depending $x_{2} g_{2}$ after SmoothSet" in Fig. 8. In this case, must_reverse at line 10 in Fig. 6 is calculated as "must_reverse (Solution 1)" in Fig. 8 and we successfully eliminate $x_{2}$ from the dependency of $g_{2}$ to get "Solution $3 "$.

Here, we consider a case where ElimVarXor fails. If we want to eliminate $x_{3}$ from the dependency of $g_{2}$ of "Solution 1" in Fig. 7, depending $x_{3} g_{2}$ at line 6 in Fig. 6 is calculated as "depending_ $x_{3} g_{2}$ after SmoothSet" in Fig. 8. In this case, ((depending $\left.x_{3} g_{2}\right)$. Consensus $\left(x_{3}\right.$, OFF $\left.\left.\left(g_{2}\right)\right)\right)$ is calculated as "obstacle" in Fig. 8. obstacle is not the constant 0 function. Therefore, there is no must_reverse, where must_reverse satisfies the "Conditions for must_reverse" and Reverse $\left(g_{2}\right.$, must_reverse) can eliminate $x_{3}$ from the dependency of $g_{2}$. This check is done at line 9 in Fig. 6. Line 8 in Fig. 6 is also needed.

There are many candidates for must_reverse that satisfy the "Conditions for must_reverse". Among them we chose $\left\{\text { depending } \__{i_{-}} g_{1}\right\}_{\bar{x}_{i}}$. If we choose another must_reverse among the candidates, we get another solution $\left(g_{1}^{\prime}, g_{2}^{\prime}\right)$. However, the variables which $g_{1}^{\prime}$ and $g_{2}^{\prime}$ depend on are the same for all candidates. Therefore, DecompXOR can find one of the existing optimal solutions even though we choose $\left\{\text { depending } x_{i_{-}} g_{1}\right\}_{\bar{x}_{i}}$ among the many candidates for must_reverse. (The proof is omitted due to space limitations.) Like DecompAND, DecompXOR can find one of the existing optimal solutions by elimination of any order of variables. (The proof is also omitted due to space limitations.)

\section{Experimental Results}

We know the methods presented here find optimal bidecomposition forms of logic functions. However, it is not certain that the methods can produce good circuits in reasonable time. Therefore, we performed a preliminary experiment on MCNC [10] benchmark circuits. The experiment was to generate a network with two-input nodes as [9] to compare the proposed methods with the other bi-decomposition method proposed in [9]. For each output function of a benchmark circuit, we decomposed the function recursively to two-input functions. The decomposition of function $f$ was done as follows:

- Find an optimal AND-Decomposition of $f$.

- Find an optimal AND-Decomposition of $\bar{f}$. (This means an OR-Decomposition of $f$.)

- Find an optimal XOR-Decomposition of $f$.

- Select the best decomposition among the above three.

- If there is no non-trivial bi-decomposition of $f, f$ is decomposed by Shannon Expansion.

Table I shows a comparison of our results and other results. The other results were taken from [9] and generated by MIS [1]. (The network levels were not reported in [9], therefore, we produced networks by MIS to examine the network levels. The script of MIS was the same as in [9].) In Table I, "nodes", "lev" and "CPU" show the number of two-input nodes, the network levels and the CPU run-time (sec.) on a Sun Ultra 22200 , respectively. "ratio" shows the ratios of the results of [9] and our method to those of MIS.

Although our results were produced without the multiple use of internal nodes, unlike [9] and MIS, the number of nodes is almost the same. Moreover, as for the network levels, the networks obtained by our method had fewer levels than those obtained by MIS.

In the experiment, our method could not find nontrivial bi-decompositions in some cases. In other words, for some functions, we cannot find a decomposition form: $f(X)=\alpha\left(g_{1}\left(X^{1}\right), g_{2}\left(X^{2}\right)\right)$ where the number of variables 
TABLE I

Decomposition to Two-Input Nodes

\begin{tabular}{|r|r|r|r|r|r|r|}
\hline Circuits & {$[9]$} & \multicolumn{2}{|c|}{ MIS } & \multicolumn{3}{|c|}{ Ours } \\
\cline { 2 - 7 } & nodes & nodes & lev & nodes & lev & CPU \\
\hline 5xp1 & 73 & 100 & 22 & 77 & 7 & 0.28 \\
\hline 9symml & 199 & 243 & 14 & 202 & 10 & 1.04 \\
\hline con1 & 15 & 17 & 4 & 16 & 4 & 0.03 \\
\hline duke2 & 550 & 641 & 26 & 759 & 11 & 30.80 \\
\hline e64 & 390 & 877 & 11 & 655 & 7 & 5.53 \\
\hline f51m & 60 & 99 & 26 & 65 & 6 & 0.12 \\
\hline misex1 & 56 & 42 & 12 & 74 & 6 & 0.11 \\
\hline misex2 & 109 & 127 & 8 & 158 & 5 & 0.16 \\
\hline misex3c & 757 & 457 & 45 & 820 & 18 & 78.36 \\
\hline rd53 & 21 & 32 & 9 & 30 & 5 & 0.05 \\
\hline rd73 & 64 & 93 & 15 & 100 & 8 & 0.32 \\
\hline rd84 & 80 & 169 & 18 & 172 & 10 & 0.70 \\
\hline sao2 & 117 & 144 & 28 & 158 & 10 & 1.01 \\
\hline z4ml & 16 & 46 & 13 & 69 & 9 & 0.18 \\
\hline total & 2507 & 3087 & 251 & 3355 & 116 & - \\
\hline ratio & 0.81 & 1.00 & 1.00 & 1.08 & 0.46 & - \\
\hline
\end{tabular}

in $X_{1}$ and that in $X_{2}$ are both less than the number in $X$. We cannot decompose such functions by bidecomposition. In the experiment, therefore, we only used Shannon Expansion for such functions. Unfortunately, we think this use of Shannon Expansion made our results worse. In such cases we must use another decomposition method, such as general decomposition: $f=\alpha\left(\vec{g}\left(X^{B}\right), X^{F}\right)$.

In the proposed methods, we do not share internal nodes among several functions. We think this was another reason causing our results to be worse. Therefore, we must combine the proposed methods with the multiple use of internal nodes. We believe the sharing of internal nodes is not so difficult. For example, we can think of the following two strategies.

- After all decompositions, we can check whether a node can be replaced with another node by the method proposed in [11].

- When $f$ is decomposed to $\alpha\left(g_{1}\left(X^{1}\right), g_{2}\left(X^{2}\right)\right)$, we can check whether an existing function can be used as $g_{1}$ (or $g_{2}$ ) by the boolean resubstitution and the support minimization technique proposed in [5].

\section{CONCLusion And Future Work}

We have presented new efficient methods to find "optimal" non-disjoint bi-decomposition AND and XOR forms. Our methods have the following properties.

- They can decompose incompletely specified functions.

- They eliminate variables one by one from the dependency of $g_{1}$ and $g_{2}$ of an intermediate solution.

- The Branch-and-Bound algorithm is used to find an optimal solution.
In this paper, a decomposition: $f(X)=\alpha\left(g_{1}\left(X^{1}\right), g_{2}\left(X^{2}\right)\right)$ is "optimal" if the total number of variables in $X^{1}$ and $X^{2}$ is the smallest. Of course, there is no guarantee that the final decomposed networks are optimal even if we adopt "optimal" decompositions at intermediate decompositions. We think, however, our meaning of "optimal" is adequate if we want to decompose functions to LUTs.

There are some functions that do not have nontrivial bi-decompositions. These functions cannot be decomposed by bi-decomposition; in the experiment, only Shannon Expansion was used for them, which is not such a good strategy. Therefore, we must develop the proposed methods by combining them with other decomposition methods. According to the experimental results, we think the proposed methods decompose functions to networks with few levels. Therefore, we plan to utilize the proposed methods to generate initial LUT networks with few levels. At this time, we do not share internal nodes among several functions in the proposed methods. Therefore, we have yet to combine the proposed methods with the multiple use of internal nodes.

\section{REFERENCES}

[1] R. K. Brayton, R. Rudell, A. Sangiovanni-Vincentelli, and A. R. Wang, "MIS: a multiple-level logic optimization system," IEEE Trans. CAD, vol. CAD-6, pp. 1062-1081, Nov. 1987.

[2] G. Lee, D. Bang, and J. Saul, "Synthesis of LUT-type FPGAs using AND/OR/EXOR representations," in Proc. SASIMI, pp. 74-77, Nov. 1996.

[3] Y. Matsunaga, "An attempt to factor logic functions using exclusive-or decomposition," in Proc. SASIMI, pp. 78-83, Nov. 1996.

[4] R. L. Ashenhurst, "The decomposition of switching functions," in Proceedings of an International Symposium on the Theory of Switching, pp. 74-116, Apr. 1957.

[5] H. Sawada, T. Suyama, and A. Nagoya, "Logic synthesis for look-up table based FPGAs using functional decomposition and support minimization," in Proc. ICCAD, pp. 353-358, Nov. 1995.

[6] J. P. Roth and R. M. Karp, "Minimization over boolean graphs," IBM journal, pp. 227-238, Apr. 1962.

[7] T. Sasao and J. T. Butler, "On bi-decompositions of logic functions," in Notes of International Workshop on Logic Synthesis (IWLS'g7), May 1997.

[8] D. Bochmann, F. Dresig, and B. Steinbach, "A new decomposition method for multilevel circuit design," in Proc. EDAC, pp. 374-377, Feb. 1991.

[9] B. Steinbach and A. Wereszczynski, "Synthesis of multilevel circuits using EXOR-gates," in Proc. of Reed-Muller'95, pp. 161-168, Aug. 1995.

[10] S. Yang, "Logic synthesis and optimization benchmarks user guide version 3.0," $M C N C$, Jan. 1991.

[11] S. Yamashita, H. Sawada, and A. Nagoya, "A new method to express functional permissibilities for LUT based FPGAs and its applications," in Proc. ICCAD, pp. 254-261, Nov. 1996. 